\title{
Performance Analysis and Simulation of Look-up Table Predistortion Algorithm
}

\author{
Qu Xiaoxu ${ }^{a}$, Lou Jingyi ${ }^{b}$ \\ Naval University of Engineering, Wuhan, Hubei, China \\ aconst.qq@163.com, bjingyi.lou@163.com
}

Keywords: Power amplifier; Nonlinearity; Predistortion; Look-up table.

\begin{abstract}
The performance of look-up table (LUT) predistortion system is analyzed and simulated, and three conclusions are gotten. The first is the system SNR increases with the increasing of the LUT length, and if the length of LUT increases doublely the SNR increases at least $6 \mathrm{~dB}$. And the second is the system with weaker nonlinearity characteristic PA has better system SNR. And the third is if the input signal with the characteristic of higher occurrence probability of little signal, the system SNR is finer. These conclusions are helpful to design the suitable parameter of the LUT predistortion system.
\end{abstract}

\section{Introduction}

Power amplifier (PA) is an integral part of the wireless communication system, but the inherent nonlinearity of PA will lead to signal distortion. Predistortion technology is an important branch of PA linearization technology, and the baseband predistortion technology has gained widely attention with the advantages of wide working bandwidth, low cost and outstanding performance [1-3].

In the existing researches, there are usually two kinds of predistorter (PD) model: polynomial model and lookup table (LUT) model. The polynomial model works well with those PAs which have weak nonlinearity characteristic [4-6], and its advantages include less system parameters, simple system structure, good system stability. But with the strongly nonlinear PA, polynomial model will bring about considerable modeling error. In this case, we need to select the LUT model as the PD model, namely use line segment fitting the PD characteristic approximatively [3,7-8].

The performance of the LUT predistortion algorithm is affected by many factors, and the relationship between them is complex. In this paper, the performance of the PD LUT algorithm has been analysed and emulated, and the research focuses on the effect on the system performance with the length of LUT, the nonlinearity characteritic of PA, and different input signal distribution.

\section{System model}

The transmitter with baseband predistortion function is shown in Fig. 1. There is a loop couplings signal from the output of PA, and after downconvertor and analog to digital converter, the digital baseband signal $v_{a}$ is gotten. We define the RF circuit, including upper and down converters and PA, as the function $P A(\bullet)$, and basing on the transmitted signal $v_{m}$ and feedback signal $v_{a}$, the fuction $P D(\bullet)$ is gotten, and with the joint system combing PD and PA, there is a linear relationship between the system input and output:

$$
v_{a}=P A\left(P D\left(v_{m}\right)\right)=k \bullet v_{m}, k \text { is a constant }
$$




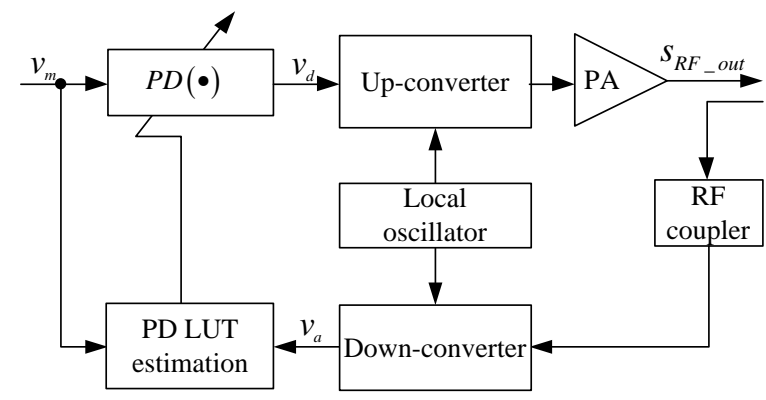

Fig. 1 The transmitter with PD

\section{Performance analysis}

Defining the input signal as $v_{m}=r_{m} e^{j \theta_{m}}$, where $r_{m}$ is the amplitude of input signal, and $\theta_{m}$ is the phase. The output signal of PA is

$$
v_{a}=r_{a} e^{j \theta_{a}}=P A\left(P D\left(v_{m}\right)\right)=r_{m} f_{a}\left(r_{m}\right) g_{a}\left(r_{m} f_{a}\left(r_{m}\right)\right) e^{j\left(\theta_{m}+f_{p}\left(r_{m}\right)+g_{p}\left(r_{m} f_{a}\left(r_{m}\right)\right)\right)}
$$

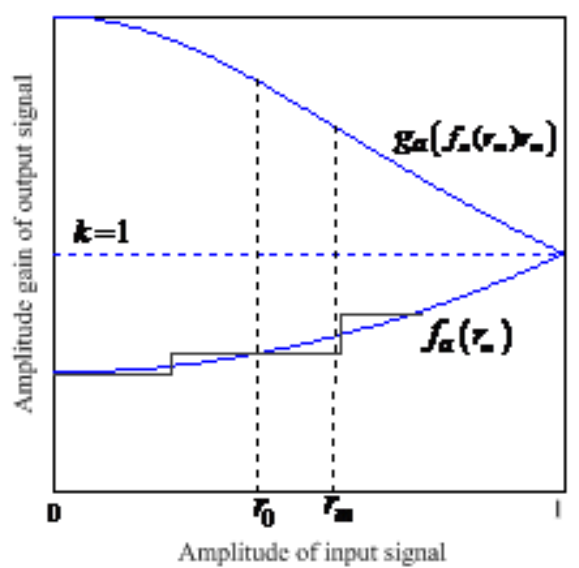

(a) Amplititude characteristic

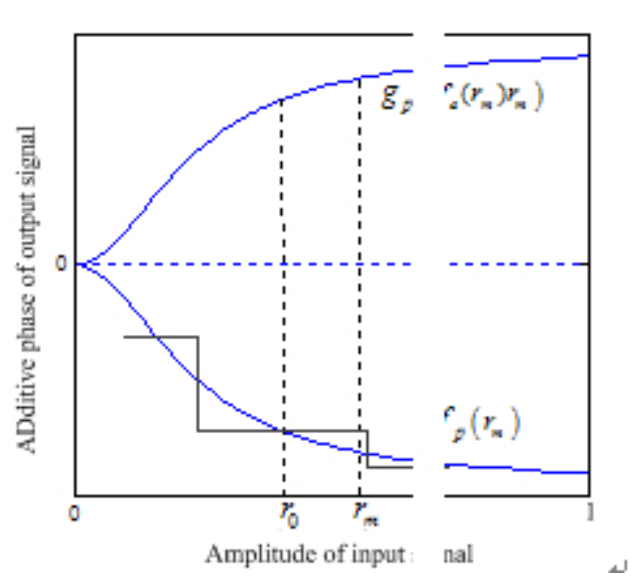

(b) Additive phase characteristic

Fig. 2 The characteristic of PD and PA

Where $f_{a}(\bullet)$ is the amplitude gain function of $\mathrm{PD}$, and $f_{p}(\bullet)$ is the additive phase shift function of PD, $g_{a}(\bullet)$ is the amplitude gain function of PA, and $g_{p}(\bullet)$ is the additive phase shift function of PA. In theory, normalize the system gain $k$, we can get

$$
\begin{aligned}
& f_{a}\left(r_{m}\right) g_{a}\left(r_{m} f_{a}\left(r_{m}\right)\right)=1 \\
& f_{p}\left(r_{m}\right)+g_{p}\left(r_{m} f_{a}\left(r_{m}\right)\right)=0
\end{aligned}
$$

In the LUT PD system, the characteristic of PD is modeled by LUT. For the limited length of LUT, quantization process is unavoidable. Fig. 2 is the characteristic diagram of PA and PD, the PD amplitude gain of the input signal with amplitude $r_{m}$ is $f_{a}\left(r_{0}\right)=f_{a}\left(r_{m}\right)+\delta f_{a}$, and the PD addative phase is $f_{p}\left(r_{0}\right)=f_{p}\left(r_{m}\right)+\delta f_{p}$. So the amplitude and phase of output signal of PA can be written as

$$
\begin{aligned}
& r_{a}=g_{a}\left(r_{m}\left(f_{a}+\delta f_{a}\right)\right)\left(f_{a}+\delta f_{a}\right) r_{m} \\
& \theta_{a}=g_{p}\left(r_{m}\left(f_{a}+\delta f_{a}\right)\right)+f_{p}\left(r_{m}\right)+\delta f_{p}+\theta_{m}
\end{aligned}
$$


Where $f_{a}$ is short for $f_{a}\left(r_{m}\right), f_{p}$ is short for $f_{p}\left(r_{m}\right)$, expand (5) and neglect the high order term of $\delta f_{a}$, we can get

$$
r_{a}=r_{m}\left(g_{a} f_{a}+r_{m} g_{a}^{\prime} f_{a} \delta f_{a}+g_{a} \delta f_{a}\right)
$$

Where $g_{a}$ is short for $g_{a}\left(r_{m} f_{a}\left(r_{m}\right)\right)$. And from Fig. 2 we know $\delta f_{a}=-\left(f_{a}\left(r_{m}\right)-f_{a}\left(r_{0}\right)\right)=-f_{a}^{\prime} \delta r_{m}$, then (7) can be written as

$$
r_{a}=r_{m}\left(g_{a} f_{a}-r_{m} g_{a}^{\prime} f_{a}^{\prime} f_{a} \delta r_{m}-g_{a} f_{a}^{\prime} \delta r_{m}\right)
$$

The derivative of (3) is

$$
r_{m} g_{a}^{\prime} f_{a}^{\prime} f_{a}+g_{a}^{\prime} f_{a}^{2}+g_{a} f_{a}^{\prime}=0
$$

Put the (3) and (9) in (8) we can get

$$
r_{a}=r_{m}\left(1+\frac{g_{a}^{\prime}}{g_{a}^{2}} \delta r_{m}\right)
$$

The same operation is done to the addative phase $\theta_{a}$, we can get

$$
\theta_{a}=k \frac{g_{p}^{\prime}}{g_{a}} \delta r_{m}+\theta_{m}
$$

Where $g_{p}$ is short for $g_{p}\left(r_{m}\left(f_{a}+\delta f_{a}\right)\right)$. The the out put signal can be written as

$$
v_{a}=r_{m}\left(1+\frac{g_{a}^{\prime}}{g_{a}^{2}} \delta r_{m}\right) e^{j\left(\frac{g_{p}^{\prime}}{g_{a}} \delta r_{m}+\theta_{m}\right)}
$$

Expanding (12) and neglect the high order term we can get

$$
v_{a}=v_{m}\left(1+\frac{g_{a}^{\prime}}{g_{a}^{2}} \delta r_{m}+j \frac{g_{p}^{\prime}}{g_{a}} \delta r_{m}\right)
$$

From (13) we know that there is systen error causing by the quantization of PD LUT, that is $v_{e}=v_{a}-v_{m}$. Deifine $\delta r_{m}=r_{m}-r_{0}$, and the signal to quantization noise ration (SNR) is

$$
S N R=\frac{P_{v_{a}}}{P_{a e}}=\frac{E\left[\left|v_{m}\right|^{2}\right]}{E\left[\left|v_{e}\right|^{2}\right]}=\int_{0}^{1} r_{m}{ }^{2} p\left(r_{m}\right) d r_{m} / \int_{0}^{1} r_{m}{ }^{2}\left[\frac{g_{a}^{\prime 2}}{g_{a}^{4}}+\frac{g_{p}^{\prime 2}}{g_{a}^{2}}\right]\left|\delta r_{m}\right|^{2} p\left(r_{m}\right) d r_{m}
$$

In the quantization process, $\delta r_{m}=2^{-N}$, where $2^{N}$ is the length of LUT with the coder bit length $N$. And from the mean value theorem for integrals, we can get 


$$
S N R \geq 2^{2 N}\left(\frac{g_{a}^{\prime 2}}{g_{a}^{4}}+\frac{g_{p}^{\prime 2}}{g_{a}^{2}}\right)_{\max }^{-1}
$$

From (14) and (15) the conclusions can be gotten that the SNR of the transmitter with LUT PD is determined by the nonlinearity character of PA, including the amplitude nonlinearity $g_{a}$ and phase nonlinearity $g_{p}$, and the distribution of the input signal $p\left(r_{m}\right)$, and the length of LUT. The increasing of the SNR with the incrasing of length of LUT following the rule that the length of coder bit $N$ increases to $N+1$, the SNR increases at least $6 \mathrm{~dB}$.

\section{Simulations}

In following simulations, the PA model is saleh model [9], and the performance of PD system is scaled by system SNR defined as

$$
S N R(d B)=10 \log _{10}\left[\sum_{n=0}^{N-1}\left|v_{m}\right|^{2} / \sum_{n=0}^{N-1}\left|v_{e}\right|^{2}\right]
$$

Fig. 3 is the relationship diagram of length of LUT and the system SNR. With the uniform distribution input signal, the SNR increases with the increasing of length of LUT, and the trend as our expectation is SNR inreases at least $6 \mathrm{~dB}$ when the LUT length increases doubly.

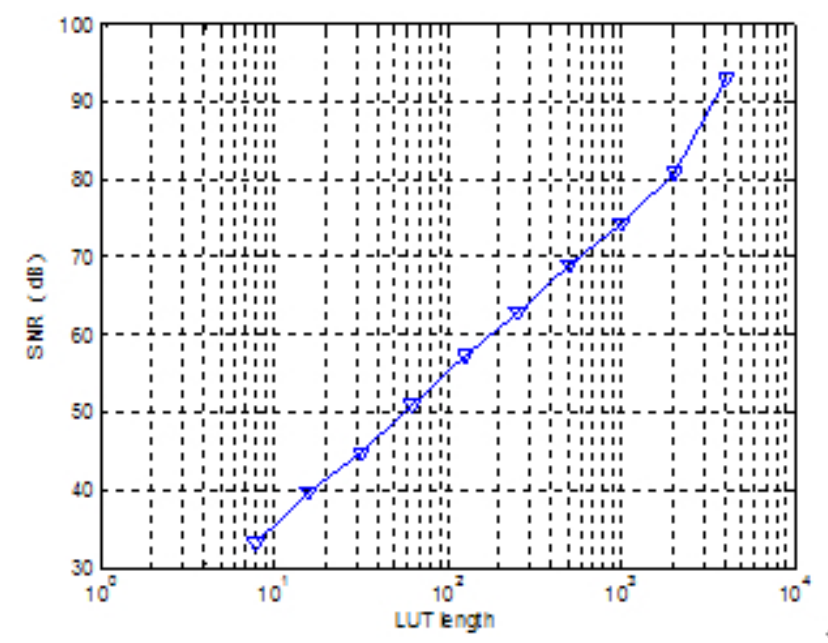

Fig. 3 Relationship of SNR with different length

of LUT

Fig. 4 is the learning curves of the PD systems with different PAs. Using the saleh model as PA model as follows

$$
g_{a}(r)=\frac{\left(1+a_{1}\right) r}{1+a_{1} \cdot r^{2}}, \quad g_{p}(r)=\frac{\pi}{12} \frac{\left(1+a_{2}\right) r^{2}}{1+a_{2} \cdot r^{2}}
$$

And three groups of values as $a_{1}=a_{2}=0.2, a_{1}=a_{2}=0.5$ and $a_{1}=a_{2}=1$ define three different nonlinearity characteristic PAs, those are PA1, PA2 and PA3. And with Nagata arithmetic [10], one of the adaptive PD LUT arithmetic, and the LUT length is 64, the conclusion is that the PA with weak nonlinearity charateristic has good system performance, the reason is that the weak nonlinearity PA comes to weak nonlinearity PD, which has slow variety nonlinearity characteric line due to little PD LUT quantization noise.

Fig. 5 shows the relationship of system SNR with different input signal distribution, including uniform distribution and OFDM signal. With same input backoff value, the OFDM signal has good 
performance, and the reason is that OFDM signal has higher occurrence probability of little signal than the uniform distribution, and the little signal has weaker nonlinearity and smaller distortion than the large one.

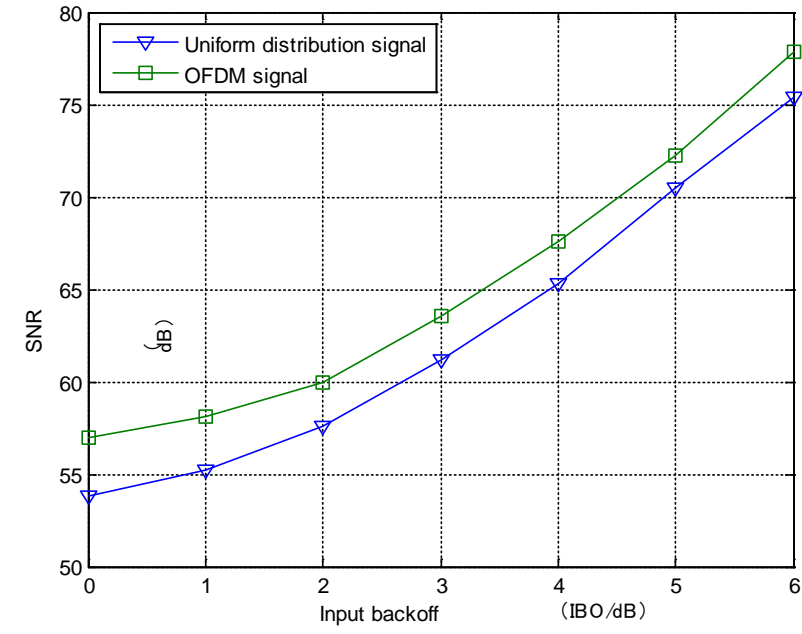

Fig. 4 The system SNR with different PAs

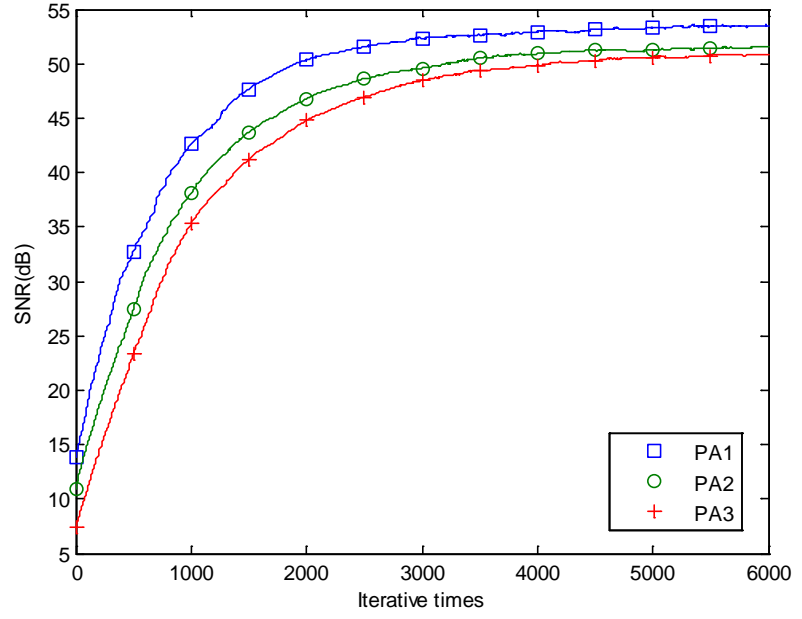

Fig. 5 The system SNR with different input signals

\section{Conclusion}

Theoretical investigation and simulation have been done on the LUT PD system, the results show that ignoring the effect of measurement noise, the system SNR is decided by the length of LUT, the nonlinearity characteristic of PA and the distribution of input signal together. Fristly, the SNR increases with the increasing of the LUT length, and if the length of LUT increases doublely the SNR increases at least $6 \mathrm{~dB}$. Secondly, the weaker nonlinearity characteristic PA comes to good system performance. And lastly, the input signal with higher exiting probability of little signal has finer SNR. These conclusions are helpful to make correct trade-off between system implementation complexities with system performance, and design the suitable parameter of the LUT predistortion system.

\section{References}

[1] R. W. Santucci, M. K. Banava: Energy-Efficient Distributed Estimation by Utilizing a Nonlinear Amplifier, IEEE Transactions on Circuits and Systems, Vol. 61(2014), p.302-311.

[2] R. Meenakshi, R. Karun: Generalized Rational Functions for Reduced-Complexity Behavioral Modeling and Digital Predistortion of Broadband Wireless Transmitters, IEEE Transactions on Instrumentation and Measurement, Vol. 2014, 63(2), p. 485-498.

[3] X. X. Qu, J. Y. Lou, J. Gao: Adaptive predistortion algorithm with optimum look-up table spacing, Journal of Huazhong University of science and technology, Vol. 40 (2012), p. 6-11.

[4] Y. J. Liu, J. Zhou, W. H. Chen: A Robust Augmented Complexity-Reduced Generalized Memory Polynomial for Wideband RF Power Amplifiers, IEEE Transactions on Industrial Electronics, Vol. 61(2014), p. 2389-2401.

[5] G. Tomas, G. Baudoin, A. Mbaye: Optimal Order Estimation for Modeling and Predistortion of Power Amplifiers, IEEE International Conference, Noisy Le Grand, France (2013).

[6] G. Baudoin, P. Jardin: Adaptive polynomial predistortion for linearization of power amplifiers in wireless communications and WLAN, International Conference on Trends in Communications, Noisy Le Grand, France(2001). 
[7] Z. Gao, P. Gui. A look-up-table digital predistortion technique for high-voltage power amplifiers in ultrasonic applications, IEEE Transactions on Ultrasonics, Ferroelectrics, and Frequency Control, Vol. 59(2012), p. 1550-1557.

[8] J. Pascale, B. Geneviève. Filter Lookup Table Method for Power Amplifier Linearization, IEEE Transactions on Vehicular Technology, Vol. 56(2007), p. 1076-1087.

[9] A. A. M. Saleh. Frequency-independent and frequency-dependent nonlinear models of TWT amplifiers, IEEE Transactions on Communications, Vol. 29(1981), p. 1715-1720.

[10] Y. Nagata, Linear amplification technique for digital mobile communications, Vehicular Technology Conference, Kawasaki-City, Japan (1989). 